Document de Recherche du Laboratoire d'Économie d'Orléans

Working Paper Series, Economic Research Department of the University of Orléans (LEO), France

DR LEO 2019-08

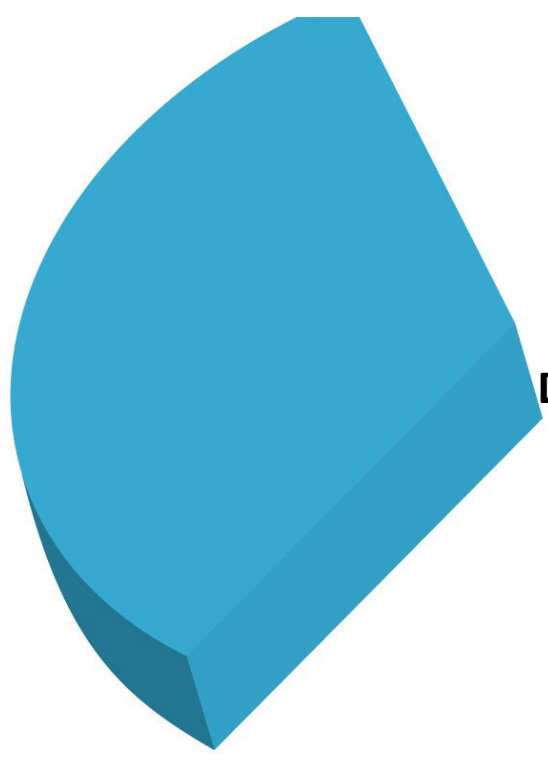

Do High-frequency-based Measures Improve

Conditional Covariance Forecasts?

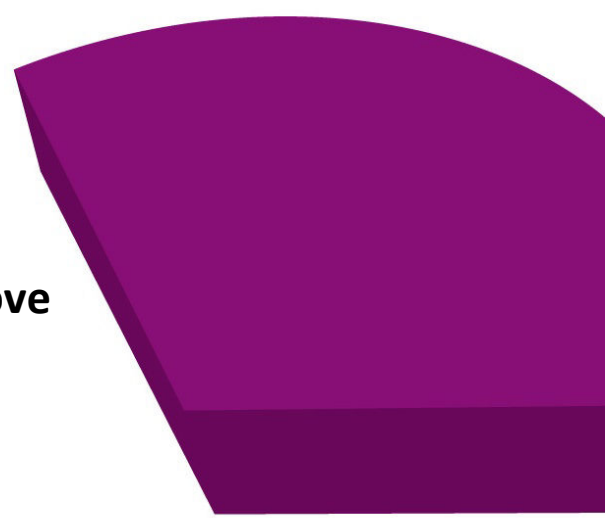

Denisa BANULESCU-RADU

Elena DUMITRESCU

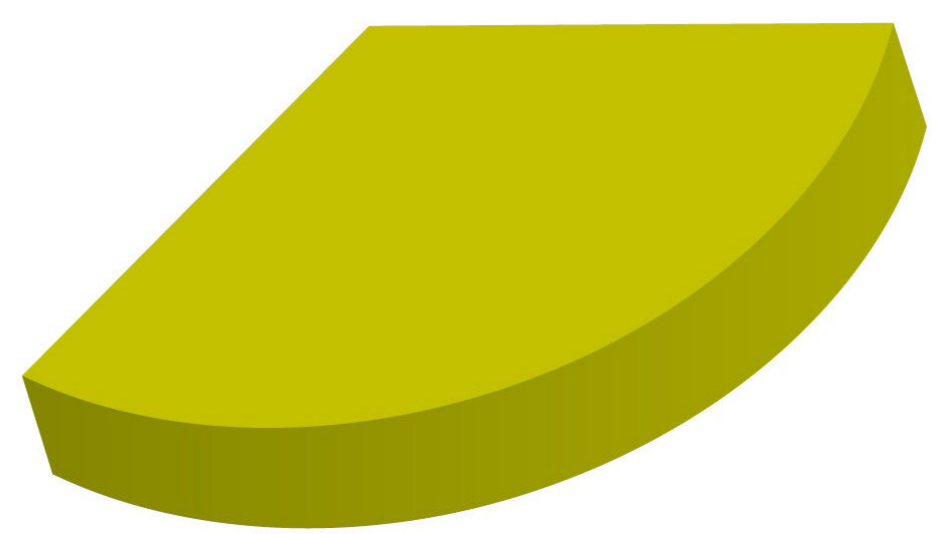

Mise en ligne / Online : 14/11/2019 


\title{
Do High-frequency-based Measures Improve Conditional Covariance Forecasts?
}

\author{
Denisa Banulescu-Radu, Elena Dumitrescu ${ }^{\dagger}$
}

June 27, 2018

\begin{abstract}
In this paper we investigate the possible benefits from using ex-post highfrequency based (realized) measures of volatility and correlation in conditional covariance forecasting. For this, we combine the (Robust) Realized GARCH framework with time varying conditional copulas and compare their forecasting abilities with those of multivariate Realized GARCH models and wellestablished competing models from the literature, i.e. the GJR-GARCH copula and the corrected DCC. The one-step-ahead forecasting abilities of the models are assessed in an empirical illustration on three pairs of financial assets by relying on the Model Confidence Set test. Our findings indicate that the proposed specifications relying on realized measures significantly improve the quality of covariance matrix forecasts.
\end{abstract}

JEL Codes: C32, C53, C58

Keywords: Covariance, Forecasting, Realized measures, Copula

*denisa.banulescu-radu@univ-orleans.fr, LEO, University of Orléans, CNRS, 45067 Orléans, France.

${ }^{\dagger}$ elena.dumitrescu@parisnanterre.fr, EconomiX, UPL, Univ. Paris Nanterre, CNRS, 92000 Nanterre, France. 


\section{Introduction}

In this paper we investigate conditional volatility and covariance forecasting by making use of ex-post high-frequency-based measures of volatility and correlation.

Although volatility is unobservable, it is a particularly relevant element in financial market analyses such as option pricing and risk management. To construct precise ex-post proxies of volatility econometricians exploit high-frequency financial data. This method has been popularized as the realized volatility approach, and it provides realized measures of volatility that are more informative about the current level of volatility than the squared returns, and are hence expected to improve volatility modeling and the accuracy of its forecasts. This part of the literature is devoted to volatility measurement and it can be distinguished from volatility prediction that is basically a reduced-form or model-based approach. The main idea is to use high-frequency data to compute ex-post realized measures of volatility at a lower frequency. The econometrics of realized volatility was first explored in Andersen and Bollerslev (1998), Andersen et al. (2001, 2003), Barndorff-Nielsen and Shephard (2002), Dacorogna et al. (2001), inter alios. The first realized measure of volatility was introduced by Andersen and Bollerslev (1998) and it is known as the realized variance (RV). The properties of this estimator are studied by Andersen et al. (2001, 2003) and Barndorff-Nielsen and Shephard (2002) who show that it is a conditionally unbiased estimator of the true daily conditional variance of the equity returns.

However, very high-frequency data may be polluted by jumps and market microstructure noise that induces autocorrelation in the intraday returns and makes the realized variance prone to a well-known bias problem. This issue was investigated in greater details by Andreou and Ghysels (2002), Bai et al. (2004), Aït-Sahalia et al. (2005) and Griffin and Oomen (2011). In the meanwhile, this sensitivity of the realized variance has motivated the development of a large number of improved 
proxies of volatility. We can mention the power and bipower variation introduced by Barndorff-Nielsen and Shephard (2004), the two-scale and multi-scale estimator by Zhang (2006), the median realized variance introduced by Andersen et al. (2012), the realized kernel by Barndorff-Nielsen et al. (2008), the intraday range- and quantilebased estimators by Christensen and Podolskij (2007) and Christensen et al. (2010). ${ }^{1}$

The research on volatility prediction has also massively evolved during the last decades and its particularity consists mainly in proposing volatility forecasting models that make extensive use of realized measures. A first approach includes the Heterogeneous Autoregressive (HAR) family of models, that produce realized volatility forecasts by using only information from the realized measures dynamics (see Corsi, 2009, among others). Second, model-based approaches consist in augmented GARCH models, i.e. that combine asset return information with realized measures so as to output conditional volatility forecasts. The GARCH-X model of Engle (2002) was the first one to be proposed, but it is an "incomplete" model in the sense that it does not model the dynamics of the realized volatility and hence it cannot produce multi-period ahead forecasts. A complete framework that jointly models returns and realized measures, i.e., Multiplicative Error Model - MEM, was introduced by Engle and Gallo (2006) and its structure was simplified by Shephard and Sheppard (2010) in their HEAVY models. However, these models operate with multiple latent volatility processes. In contrast, the Realized GARCH (henceforth RGARCH) framework by Hansen et al. (2012) relies on a so-called measurement equation to relate the realized measure to the underlying conditional variance. Hansen and Huang (2016) and Lunde and Olesen (2013) find that RGARCH models significantly improve volatility forecasts. Extensions to a multivariate setup including financial assets or exchange rates are also found to provide good empirical fit for the data under analysis (see Hansen et al., 2014; Dumitrescu and Hansen, 2018).

\footnotetext{
${ }^{1}$ See also Andersen et al. (2009), Hansen and Horel (2009) and references therein.
} 
Nevertheless, when a large set of assets is to be analyzed, all volatility forecasting approaches with or without realized measures suffer from the curse of dimensionality. Various hypotheses have been made in the literature so as to be able to model large financial systems, see e.g. Engle (2002); Engle and Kroner (1995) in the GARCH framework and Chiriac and Voev (2011); Callot et al. (2017); Hansen et al. (2014) in frameworks using realized measures.

A simpler, flexible and more parsimonious way to model high-dimensional systems is through the use of copula-based models. Indeed, copulas can be used to construct high-dimension distributions with specified dependence and arbitrary marginal distributions, see Patton (2012) for a review. Based on Sklar (1959)'s theorem extended to conditional distributions by Patton (2006), the researcher can model the marginal distributions separately from the conditional one (here a conditional copula). The major advantages of this approach are its flexibility beyond existing multivariate distributions and the simplicity of implementation of two-stages estimation that reduces computational burden.

In this paper we investigate the possible benefits from using realized measures in conditional covariance forecasting. For this, on the one hand we propose to model large systems of financial assets by combining the advantages of the RGARCH framework, that will be used to model the conditional marginal distributions of the assets, and those of time varying conditional copulas so as to form a dynamic conditional joint distribution. We henceafter label this approach the Realized GARCH copula model (i.e. RGARCH copula). On the other hand, we estimate multivariate realized GARCH models similar to those proposed by Dumitrescu and Hansen (2018). The forecasting abilities of these two types of models relatively to those of traditional models, that do not exploit the information included in high-frequency financial data, are assessed by relying on the Model Confidence Set (MCS) approach of Hansen et al. (2011) with robust loss functions à la Patton (2011). 
We illustrate the usefulness of the realized measures for conditional covariance forecasting in an empirical application on three pairs of SP500 companies exhibiting different levels of daily return correlation and covering different sectors: Citigroup JP Morgan, Boeing - Procter\&Gamble, and Pepsi - Coca-Cola.

We analyze the one-period ahead forecasting abilities of the RGARCH copula and the bivariate RGARCH models relatively to those of well-established competing models from the literature: the GJR-GARCH copula of Patton (2013) and the corrected-DCC model by Aielli (2013). We also include in this horse race the RobustRGARCH version of the proposed model by drawing on Banulescu-Radu et al. (2018). We find that significant gains in conditional covariance forecasting emerge from the use of ex-post high-frequency-based measures of volatility and correlation. The role that realized measures of volatility and correlation play in conditional covariance forecasting should therefore receive more attention in larger-scale empirical forecasting analyses.

The rest of the paper is organized as follows. Section 2 introduces the copulabased models and the multivariate RGARCH ones, while Section 3 presents the conditional covariance forecasting approach. The results of the empirical analysis are revealed in Section 4, and Section 5 concludes.

\section{Methodology}

In this section we first introduce briefly the main realized measures of variance and correlation. Then, we detail the multivariate models compared that include realized measures of variance and/or correlation: i) the RGARCH Copula models, and ii) the bivariate RGARCH models. ${ }^{2}$

\footnotetext{
${ }^{2}$ The traditional DCC-type models are not detailed here. For a review on them see Bauwens et al. (2012), and Aielli (2013) for the specificities of the cDCC model.
} 


\subsection{Realized measures of variance and correlation}

The realized measures of volatility rely on the continuous-time volatility process or the evolution of the spot volatility (i.e. instantaneous volatility). Nevertheless, volatility measurements are generally restricted to discrete-time intervals. By integrating the spot volatility over a daily horizon, for instance, one obtains the ex-post daily integrated variance (IV). In presence of jumps, the natural target for realized volatility measurement is the quadratic variation $(\mathrm{QV})$, which extends the definition of the integrated volatility by including also the cumulative squared jumps.

To set notations, let $p_{t}$ denote the logarithmic price for a financial asset sampled at daily frequency $t$, and the corresponding daily return be defined by $r_{t, t-1} \equiv \log \left(p_{t}\right)-$ $\log \left(p_{t-1}\right)$. Equally spaced series of continuously compounded returns are assumed to be observed $m$ times per day (or to have an intraday sampling frequency $\tau=1 / m$ ), and be computed as $r_{t, i}^{(\tau)}=p_{t, i / m}-p_{t,(i-1) / m}$.

In the following, we limit our attention to three commonly used realized measures of volatility, and introduce first the so-called realized variance estimator (RV) proposed by Andersen and Bollerslev (1998). This estimator is simply obtained by summing up the squared intraday returns over a day $t$. More formally, it is defined as follows:

$$
R V_{t}^{(\tau)}=\sum_{j=1}^{m} r_{t, j}^{(\tau) 2}
$$

Under suitable conditions (such as the absence of serial correlation in the intraday returns) it can be shown that $R V_{t}^{(\tau)}$ converges to the so-called integrated variance as $m \rightarrow \infty$. However, in the presence of jumps, the realized volatility is not a consistent estimator of the integrated volatility anymore. Several estimators of IV robust to jumps have been developed. One such consistent measure of the integrated variance is the bipower variation (BPV) pioneered by Barndorff-Nielsen and Shephard (2004) 
and defined as:

$$
B P V_{t}^{(\tau)}=\mu_{1}^{-2} \frac{m}{m-1} \sum_{j=2}^{m}\left|r_{t, j}^{(\tau)}\right|\left|r_{t, j-1}^{(\tau)}\right|,
$$

where $\mu_{1} \equiv \sqrt{2 / \pi} \approx 0.79788$. Unlike the RV, BPV is designed to be robust to jumps by construction, i.e. it is obtained by summing up the product of two consecutive returns instead of the squared return, which means that a jump occurring at time $t$ will be multiplied by a very small return observed at time $t+1 .^{3}$

However, in practice, at very high-frequencies, returns are polluted by microstructure noise (e.g., bid-ask bounce, unevenly spaced observations, discreteness, etc.), which induces autocorrelation in the high-frequency returns. To counteract the adverse effects of microstructure noise, the price process is often sampled at 1,5 or 30 minutes, the 5 minutes frequency arising as the optimal one in many empirical applications, see e.g. Liu et al. (2015); Banulescu-Radu et al. (2016). At the same time, motivated by the specificities of the intraday financial time series (namely the presence of microstructure noise), researchers have proposed improved estimators of the quadratic variation. A benchmark class of estimators is given by the so-called realized kernel estimators, who provide a simple solution to the problem of estimating the long-run variance of a process observed at discrete times. In particular, a consistent measure of QV in the presence of second-order stationary noise is the realized

\footnotetext{
${ }^{3}$ Other well known alternatives to the BPV include the median realized variance (medRV) proposed by Andersen et al. (2012) and computed as the sum of the squared median of three consecutive intraday returns, the quantile-based realized variance (QRV) of Christensen et al. (2010), the "nearest neighbor truncation" estimators of Andersen et al. (2012), i.e. the "MinRV" and "MedRV" estimators, and the truncated or threshold realized variance of Mancini $(2001,2009)$.
} 
kernel (RK) estimator by Barndorff-Nielsen et al. (2008): ${ }^{4}$

$$
R K_{t}^{(\tau)}=\sum_{h=-H}^{H} k\left(\frac{h}{H+1}\right) \gamma_{h, t}
$$

where $\gamma_{h, t}=\sum_{j=|h|+1}^{m} r_{t, j}^{(\tau)} r_{t, j-|h|}^{(\tau)}, \gamma_{h, t}=\gamma_{-h, t}, k($.$) is the Parzen kernel function and$ $H$ is a bandwidth determined by following the recommendations of Barndorff-Nielsen et al. (2009).

In the sequel of this chapter we rely on the multivariate versions of the realized variance and the realized kernel estimators. The realized quadratic covariation (hereafter realized covariance estimator, RCov) by Andersen et al. (2003) is an immediate extension of the RV measure:

$$
\operatorname{Rov}_{t}^{(\tau)}=\sum_{j=1}^{m} \mathbf{r}_{t, j}^{(\tau)} \mathbf{r}_{t, j}^{(\tau) \prime}
$$

where $\mathbf{r}_{t}(\tau)$ is an $n$-dimensional return vector observed at time $\tau$ on day $t$. At the same time, the multivariate extension of the realized kernel approach (hereafter, $\left.M R K_{t}^{(\tau)}\right)$ arises by feeding the $n$-dimensional return vector $\mathbf{r}_{t}^{(\tau)}=\left[r_{1, t}^{(\tau)}, r_{2, t}^{(\tau)}, \ldots, r_{n, t}^{(\tau)}\right]^{\prime}$ to $\gamma_{h, t}$ in $(3)$.

The corresponding realized measures of correlation can be subsequently derived

\footnotetext{
${ }^{4}$ Several other estimators of QV based on high-frequency data that correct for microstructure noise, and which under certain conditions on the market microstructure noise are consistent at the optimal rate, have been proposed in the literature, e.g. the two-scale realized variance (TSRV) of Zhang et al. (2005), the multi-scale realized variance (MSRV) of Zhang (2006), the pre-averaged realized variances estimator by Podolskij and Vetter (2009) and Jacod et al. (2009), the "realized range-based variance" (RRV) of Christensen and Podolskij (2007) and Martens and Van Dijk (2007), the maximum likelihood Realized Variance of Aït-Sahalia et al. (2005), etc., but their analysis is out of the scope of this paper.
} 
from the realized covariance matrices:

$$
R \operatorname{Corr}_{i j, t}=\frac{R \operatorname{Cov}_{i j, t}^{(\tau)}}{\sqrt{R V_{i, t}^{(\tau)} R V_{j, t}^{(\tau)}}} \text { or } R K \operatorname{Corr}_{i j, t}=\frac{M R K_{i j, t}^{(\tau)}}{\sqrt{R K_{i, t}^{(\tau)} R K_{j, t}^{(\tau)}}} .
$$

\subsection{Multivariate models with Copula dependence}

Let $\mathbf{r}_{t}=\left[r_{1, t}, r_{2, t}, \ldots, r_{n, t}\right]^{\prime}, t=1, \ldots, T$, denote the vector of asset returns under analysis at time $t$. Its conditional distribution with respect to the information set, $\mathbf{G}\left(\cdot \mid \mathcal{F}_{t-1}\right)$, is given by $\mathbf{r}_{t} \mid \mathcal{F}_{t-1} \sim \mathbf{G}\left(\cdot \mid \mathcal{F}_{t-1}\right)$, where $\mathcal{F}_{t-1}$ denotes the information set generated by $\left(\mathbf{r}_{t-1}, \mathbf{r}_{t-2}, \ldots\right)$. As usual, the DGP for each asset returns allows for time-varying conditional mean and variance,

$$
r_{i, t}=\mu_{i, t}+\sqrt{h_{i, t}} z_{i, t}, i=1, \ldots, n,
$$

with the conditional mean, $\mu_{i, t}=\mathbb{E}\left(r_{i, t} \mid \mathcal{F}_{t-1}\right)$, often considered to be constant or zero, $h_{i, t}$ the conditional standard deviation, and where the studentized innovations, $z_{i, t}$, follow a parametric distribution of mean zero and variance one, $z_{i, t} \mid \mathcal{F}_{t-1} \sim G_{i}\left(\cdot \mid \mathcal{F}_{t-1}\right)$.

\subsubsection{Copula dependence}

Using Sklar's theorem extended to conditional joint distributions in Patton (2006), the conditional distribution of $\mathbf{r}_{t}$ given $\mathcal{F}_{t-1}$ is decomposed into the conditional marginal distributions of returns and the conditional copula that accounts for the dependence structure:

$$
\mathbf{G}\left(\mathbf{r}_{t} \mid \mathcal{F}_{t-1}\right)=\mathbf{C}\left(G_{1}\left(r_{1, t} \mid \mathcal{F}_{t-1}\right), \ldots, G_{n}\left(r_{n, t} \mid \mathcal{F}_{t-1}\right)\right)
$$

where $\mathbf{C}:[0,1]^{n} \rightarrow[0,1]$ is a $n$-copula function and $G_{1}(),. \ldots, G_{n}($.$) are the marginal$ distribution functions. 
This decomposition allows one to draw on the vast literature on modeling and forecasting conditional covariance matrices by relying either on low or on highfrequency data. At the same time, one can avoid the curse of dimensionality and computational burden by estimating the model in stages (see Subsection 2.2.3 for more details). Consequently, a major advantage of this decomposition is that the proposed model can be easily extended to high dimensions and the estimation is feasible and fast.

A variety of copula functions have been proposed to date in the statistical literature, but we recall here only the most commonly used ones in financial applications, that belong to i) the elliptical family of distributions, i.e. Gaussian and Student-t, or to ii) the Archimedian family, i.e. Gumbel and Clayton.

Also called implicit copulas, the Gaussian and Student-t copulas do not have a simple closed form, but are implied by well-known multivariate distribution functions. Indeed, the probability density functions of these copulas are easy to compute. The underlying distributions imply symmetry and different degrees of heavy-tailness for the copulas according to the degrees of the freedom parameter $\nu$. If one believes in the asymmetries of financial return dependence, such copulas could appear as too restrictive to provide a reasonable fit.

Asymmetric copula, exhibiting larger dependence in one tail than in the other, might be a better choice. This is the case of Archimedean copulas. If the Clayton puts dependence in the negative tail, the Gumbel has only positive tail dependence. A rotated version of the Gumbel is hence preferred in financial analyses where negative events tend to cluster together more than positive ones. Archimedean copulas are explicit copulas, that although are not derived from multivariate distribution functions, do have simple closed forms. They play an important role in finance because they present several desired properties: they are asymmetric, easily derived, associative and easy to estimate. Archimedean copulas are capable of capturing 
wide ranges of dependence for different choices of the generator function and they are related to multivariate distributions generated by mixtures. All these copulas and extensions thereof will be compared in the empirical application in Section 4.

Various extensions of the aforementioned copulas have been proposed in the literature so as to account for the specificities of financial data. In particular, time varying copulas, that allow the dependence parameter to vary through time, have gained a lot of attention. A nice way to specify the dynamics of the dependence parameter is by relying on realized measures of correlation. For this reason, the mechanism of these extended copulas will be detailed in Section 2.2.2, where we introduce the proposed multivariate models that mix realized measures and copula dependence.

\subsubsection{Multivariate models with realized measures and Copula depen- dence}

The forecasting abilities of the model in (6) will depend upon the choices made for the marginal distributions as well as for the conditional copula.

The benchmark multivariate copula model in the financial literature is a constant GJR-GARCH copula one, i.e. based on the traditional GJR-GARCH for modeling the marginal distribution of each asset and constant correlation parameter, which belongs to the general class of copula-MGARCH models. By analogy with the traditional multivariate GJR-GARCH models, e.g. DCC GJR-GARCH, this specification is designed to account for leverage, i.e. stronger impact of negative shocks on volatility than that of positive shocks while specifying the joint dynamics of the return series. In the empirical illustration, in Section 4, the copula-MGARCH models obtained by combining these GJR-GARCH marginals with the various copulas described in the previous section will be compared in terms of forecasting abilities.

To this comparison we add a new class of multivariate copula models, called Real- 
ized GARCH copula, that account for the benefits from using both realized measures and copula dependence in conditional covariance forecasting. There are basically two ways to include realized measures in a multivariate copula model. The first one consists in modeling the marginal distributions of returns jointly with realized measures of volatility, as in the Realized GARCH framework (see e.g. Hansen et al., 2012). The second approach derives from the multivariate character of the model and it consists in linking the dynamics of the copula correlation parameter to a realized measure of correlation. In the latter case the model belongs to the time-varying class of copulas.

In the Realized GARCH copula we hence propose to account for realized measures in both components of the model by drawing on the works on RGARCH on the one hand and on Generalized Autoregressive Score (GAS) models by Harvey (2013) and Creal et al. (2013) on the other hand. In particular, we specify the time-varying conditional marginal distribution of each asset as a RGARCH model. Indeed, modeling the dynamics of the realized measures in relation with the conditional measures of volatility through measurement equations has been shown to improve conditional volatility estimation and forecasting relatively to traditional GARCH models (see, for instance, Hansen et al., 2012; Lunde and Olesen, 2013; Dumitrescu and Hansen, 2018). To make the RGARCH model complete, besides the returns equation in (6) we specify the GARCH and measurement equations, for which we choose a log-linear form that easily guarantees the positivity of the variance:

$$
\begin{aligned}
& \log h_{i, t}=\alpha_{i}+\beta_{i} \log h_{i, t-1}+\tau_{i}\left(z_{t-1}\right)+\gamma_{i} u_{i, t-1} \\
& \log x_{i, t}=\xi_{i}+\varphi_{i} \log h_{i, t}+\delta_{i}\left(z_{t}\right)+u_{i, t}
\end{aligned}
$$

where $\tau_{i}\left(z_{t-1}\right)=\tau_{i, 1} z_{i, t-1}+\tau_{i, 2} z_{i, t-1}^{(2)}$ is called the leverage function. Here we adopt a quadratic specification with $z_{i, t-1}^{(2)}=z_{i, t-1}^{2}-1$ for $i=1, \ldots, n$ by following Hansen et al. (2012), with $z_{i, t}=r_{i, t} / \sqrt{h_{i, t}}$. Similarly, $\delta_{i}(z)=\delta_{i, 1} z_{i, t}+\delta_{i, 2} z_{i, t}^{(2)}$. Besides, the 
measurement errors, $z_{i, t} \sim i i d(0,1)$ and $u_{i, t} \sim i i d\left(0, \sigma_{u}^{2}\right)$, are assumed to be mutually independent, in the sense that the dependence of realized measures of volatility on returns is fully captured by $\delta_{i}(z)$.

To account for unusually large shocks to returns and volatility, we also consider the robustified version of the RGARCH model proposed by Banulescu-Radu et al. (2018), hereafter RRGARCH model, as a specification for the marginals. For this extension, the authors adopted some insights from Harvey (2013) by introducing parameters that serve to dampen the impact of outliers in returns and volatility. To implement the model, one substitutes $z_{i, t}$ by $\tilde{z}_{i, t}=z_{i, t} / \sqrt{1+z_{i, t}^{2} / d_{z_{i}}}$, and $u_{i, t}$ by $\tilde{u}_{i, t}=u_{i, t} / \sqrt{1+\left(u_{i, t} / \sigma_{u_{i}}\right)^{2} / d_{u_{i}}}$ in the GARCH equation, where $d_{z_{i}}$ and $d_{u_{i}}$ are robustness parameters to be estimated. The standard RGARCH model emerges in the limit as $d_{z_{i}}, d_{u_{i}} \rightarrow \infty$.

To construct each of the parsimonious multivariate models we map returns' univariate marginal distribution, $G_{i}$, to the joint distribution, $\mathbf{G}$, through a constant or time-varying copula function such as those discussed in Section 2.2. Anticipating on the empirical results, the time varying Student-t copula, with constant degrees of freedom and time varying correlation parameter $\zeta_{t}$, appears to perform best:

$$
\mathbf{G}\left(\mathbf{r}_{t} \mid \mathcal{F}_{t-1}\right)=\mathbf{C}\left(U_{1, t}, \ldots, U_{n, t} ; \zeta_{t}\right)
$$

where $U_{i, t} \equiv G_{i}\left(r_{i, t} \mid \mathcal{F}_{t-1}\right)$ are conditional probability integral transform variables of the return data. Indeed, Patton (2013) also finds it to perform well in financial applications. The evolution of the copula parameter $\zeta_{t}$ is assumed to be governed by the "GRAS" specification (Realized Generalized Autoregressive Score) inspired by the GAS models proposed by Harvey (2013) and Creal et al. (2013), and augmented with realized measures as discussed in Salvatierra and Patton (2015). Since correlation has restricted support, we model the dynamics of a strictly increasing transformation 
of $\zeta_{t}, F\left(\zeta_{t}\right)=\frac{1}{2} \log \left(\frac{1+\zeta_{t}}{1-\zeta_{t}}\right)$, i.e. its Fisher transform, as:

$$
F\left(\zeta_{t}\right)=\omega+B F\left(\zeta_{t-1}\right)+A s_{t-1}+\vartheta F\left(y_{t-1}\right)
$$

where $F\left(y_{t}\right)$ is the Fisher transform of a generic realized measure of correlation, $y_{t}$,

and $s_{t}$ the score of the copula likelihood given by $s_{t}=S_{t} \nabla_{t}$, with $\nabla_{t}=\frac{\partial \log \mathbf{c}\left(U_{\left.1, t, \ldots, U_{n, t} ; \zeta_{t}\right)}\right.}{\partial \zeta_{t}}$. c is the copula density function and $S_{t}=\mathbf{I}^{-1 / 2}$ the (Cholesky) square root of the inverse Hessian matrix. Note also that the "GRAS" specification comes down to the "GAS" one if the dependence of the copula parameter on the realized measure of correlation is dropped, i.e. the last term in (11) vanishes.

\subsubsection{Likelihood function}

The (R)RGARCH-GRAS-copula models are simple to estimate by multi-stage quasimaximum likelihood (MSQML). Although this approach is less efficient than onestage MLE, according to Patton (2006) the loss is not great in many cases, while the estimation problem in high dimensional systems is greatly simplified. Denote by $\theta=\left[\psi, \theta_{c}\right]^{\prime}$ the parameters for the entire model, with $\psi^{\prime}=\left[\psi_{1}, \ldots, \psi_{n}\right]$ containing the parameters related to all the marginal distributions, i.e. $\psi_{i}$ includes the parameters of the (R)RGARCH model for asset $i, i=1, \ldots, n$, and $\theta_{c}$ the parameters of the copula.

The conditional joint distribution of the returns can be decomposed into the conditional marginal return distribution for each asset and the conditional copula by applying the extended version of the Sklar's theorem (Patton, 2006):

$$
\mathbf{f}\left(\mathbf{r}_{t} \mid \mathcal{F}_{t-1}\right)=\prod_{i=1}^{n} f_{i, t}\left(r_{i, t} \mid \mathcal{F}_{t-1} ; \psi_{i}\right) \times \mathbf{c}\left(G_{1}\left(r_{1, t} \mid \mathcal{F}_{t-1} ; \psi_{1}\right), \ldots, G_{n}\left(r_{n, t} \mid \mathcal{F}_{t-1} ; \psi_{n}\right) ; \theta_{c}\right)
$$

where $\mathbf{c}$ denotes the copula density function. The associated log-likelihood function 
is:

$\log \mathcal{L}(\theta)=\sum_{t=1}^{T}\left[\sum_{i=1}^{n} \log f_{i, t}\left(r_{i, t} \mid \mathcal{F}_{t-1} ; \psi_{i}\right)+\log \mathbf{c}\left(G_{1}\left(r_{1, t} \mid \mathcal{F}_{t-1} ; \psi_{1}\right), \ldots, G_{n}\left(r_{n, t} \mid \mathcal{F}_{t-1} ; \psi_{n}\right) ; \theta_{c}\right)\right]$

The model is hence specified in such a way that the parameters can be estimated in two stages. In the first stage, the parameters of each margin are estimated independently of the other margins (assets) as

$$
\widehat{\psi}_{i}=\underset{\psi_{i}}{\operatorname{argmax}} \sum_{t=1}^{T} f_{i, t}\left(r_{i, t}, x_{i, t} \mid \mathcal{F}_{t-1} ; \psi_{i}\right), i=1, \ldots, n, t=1, \ldots, T
$$

where the joint density of the RGARCH model can be rewritten as

$$
\begin{aligned}
f_{i, t}\left(r_{i, t}, x_{i, t} \mid \mathcal{F}_{t-1} ; \psi_{i}\right) & =f_{i, t}\left(r_{i, t} \mid \mathcal{F}_{t-1} ; \psi_{i}^{(1)}\right) \times f_{i, t}\left(x_{i, t} \mid r_{i, t}, \mathcal{F}_{t-1} ; \psi_{i}\right) \\
& =-\frac{1}{2} \sum_{t=1}^{T}\left[\log \left(h_{i, t}\right)+r_{i, t}^{2} / h_{i, t}+\log \left(\sigma_{u_{i}}^{2}\right)+u_{i, t}^{2} / \sigma_{u_{i}}^{2}\right]
\end{aligned}
$$

with $\psi_{i}^{(1)}$ the vector of RGARCH parameters except for $\sigma_{u_{i}}^{2}$, and the first term in the decomposition being precisely the conditional marginal return density function. The density of the RRGARGH model takes a similar form, where we account for the two transformations of the innovations and their corresponding parameters.

In the second stage, the parameters of the time-varying copula are estimated by

$$
\widehat{\theta}_{c}=\underset{\theta_{c}}{\operatorname{argmax}} \sum_{t=1}^{T} \log \mathbf{c}\left(G_{1}\left(r_{1, t} \mid \mathcal{F}_{t-1} ; \widehat{\psi}_{1}\right), \ldots, G_{n}\left(r_{n, t} \mid \mathcal{F}_{t-1} ; \widehat{\psi}_{n}\right) ; \theta_{c}\right)
$$

Following Patton (2013), we choose for $G_{i}$ the Hansen's (1994) skewed-t distribution. Combining parametric marginal distributions for the standardized residuals with parametric models for the conditional means and variances simplifies the inference procedure for the copula parameters. Under standard regularity conditions it can be 
shown that $\sqrt{T}\left(\hat{\theta}_{T}-\theta^{*}\right) \stackrel{d}{\rightarrow} N\left(0, V_{M S M L}\right)$, as $T \rightarrow \infty .^{5}$ A consistent estimator of the asymptotic covariance matrix $V_{M S M L}$ can be obtained in the form of a "sandwich" estimator so as to capture the effect of first-step estimation error on the estimation of the copula parameter. ${ }^{6}$

\subsection{Multivariate Realized GARCH}

In this section we introduce a second category of multivariate models for conditional covariance that make use of realized measures. Multivariate Realized GARCH models are simple extensions of univariate RGARCH models (see Hansen et al., 2012; Hansen and Huang, 2016). They have been shown to exhibit good forecasting abilities in the particular case of exchange rates by Dumitrescu and Hansen (2018). Similar to the univariate model, the multivariate model is structured in three sets of equations: i) equations for returns, ii) equations constituting the multivariate GARCH system, and iii) measurement equations. If the $n$ return equations are given in (6) and hence supposed common across all multivariate models under analysis, the multivariate GARCH system jointly models the dynamics of the log-conditional variances as well as the dynamics of the Fisher transformed conditional correlations:

$$
H_{t}:=\boldsymbol{\alpha}+\boldsymbol{\beta} H_{t-1}+\boldsymbol{\tau}\left(z_{t}\right)+\gamma \mathrm{w}_{t},
$$

with

$$
H_{t}=\left(\begin{array}{c}
\log \left(h_{t}\right) \\
F\left(\rho_{t}\right)
\end{array}\right), z_{t}=\left(\begin{array}{c}
z_{1, t-1} \\
\ldots \\
z_{n, t-1}
\end{array}\right), \mathrm{w}_{t}=\left(\begin{array}{c}
u_{1, t-1} \\
\ldots \\
u_{n, t-1} \\
v_{t-1}
\end{array}\right)
$$

\footnotetext{
${ }^{5}$ For more details, see White (1996), as well as the discussion in Patton (2013).

${ }^{6}$ An alternative method is to use a block bootstrap for inference, see Gonçalves and White (2004).
} 
and where $\boldsymbol{\tau}\left(z_{t}\right)=\tau_{1} z_{t-1}+\tau_{2} z_{t-1}^{(2)}$ as discussed in the univariate setup. Denote by $q=n(n+1) / 2$ the number of elements in $H_{t}$. Then scalar parameters in (8) and (9) are replaced here by vectors or matrices, but they preserve their interpretation. In particular, $\boldsymbol{\alpha}$ is a $q \times 1$ vector, $\boldsymbol{\beta}$ and $\boldsymbol{\gamma}$ are $q \times q$ matrices, while $\boldsymbol{\tau}_{\mathbf{1}}$ and $\boldsymbol{\tau}_{\mathbf{2}}$ are $q \times n$ matrices. $F\left(\rho_{t}\right)$ is the Fisher transform of the conditional correlation and the set of measurement equations accounts for the dynamics of both realized measures of variance $x_{t}=\left\{x_{1, t}, \ldots, x_{N, t}\right\}$ and correlation $y_{t}=\left\{y_{i j, t}\right\}, i \neq j, i>j, i, j \in\{1, \ldots, N\}$ :

$$
\begin{aligned}
\log \left(x_{t}\right) & =\xi+\varphi \log \left(h_{t}\right)+\delta\left(z_{t}\right)+u_{t} \\
F\left(y_{t}\right) & =\zeta+\psi F\left(\rho_{t}\right)+v_{t}
\end{aligned}
$$

Note that for parsimony reasons the measurement equations do not account for cross-assets dependence, i.e. they are designed exactly as in the univariate case. For simplicity, we further assume that the vector of measurement errors $\mathrm{u}_{t-1}=$ $\left(u_{1, t-1}, u_{2, t-1}, v_{t-1}\right)^{\prime} \sim \mathcal{N}_{n(n+1) / 2}(0, \Omega)$, i.e. it follows a multivariate normal distribution with zero-mean and covariance matrix $\Omega$ :

$$
\Omega=\left(\begin{array}{cc}
\Omega_{11} & \Omega_{12} \\
\cdot & \Omega_{22}
\end{array}\right),
$$

where the three submatrices correspond to the covariances of the innovations in (14), (15) and cross covariances of all these innovations, respectively.

The conditional measures in (13) follow a vector autoregressive process of order one, VAR(1) with multiple sources of dynamics. First, the $\boldsymbol{\beta}$ matrix accounts for traditional interdependence between the conditional covariance elements in the form of persistence (on the main diagonal) and spillovers (off-diagonal). Its diagonal elements are subject to standard stationarity constraints. Second, through the leverage function, $\boldsymbol{\tau}($.$) , the lagged studentized returns can impact conditional covariance ele-$ 
ments in an asymmetric way. Besides, the lagged measurement errors can drive the conditional covariance dynamics up and down through the $\gamma$ matrix.

The multivariate Realized GARCH model can be easily estimated by QMLE by following the same steps as for the univariate RGARCH (see Dumitrescu and Hansen, 2018, for more details). However, this model suffers from the curse of dimensionality when $n$ increases. To make it more parsimonious, constraints such as diagonalizing the matrix $\boldsymbol{\beta}$ and / or $\boldsymbol{\gamma}$ in (13) can be imposed to the full model. This suggestion, that we will refer to as the diagonal bivariate RGARCH model, will be taken into account in the empirical analysis.

\section{Forecasting}

The fully parametric structure of the various models compared makes it easy to forecast the covariance matrix one-period ahead.

In the case of the Copula-dependence models, the variance and covariance terms are forecasted separately based on the specifications chosen for the marginals (GJRGARCH, RGARCH or RRGARCH) and for the time-varying copula (with "GAS" or "GRAS" correlation dynamics), respectively. On the one hand, by iterating forward on (8) for each asset and using the exponential transform, one immediately computes the one-step-ahead variances. ${ }^{7}$ On the other hand, the computation of the correlation and implicitly that of the covariance has to be done in two steps. First, iterating on (11) in a similar way as for the marginals, followed by the inverse Fisher transform of the outcome, reveals the one-period ahead time-varying correlation parameter of the Student copula. To extract the level of linear correlation from the copula, we then rely on the simulation approximation proposed by Patton (2013) with 20 nodes and 50,000 repetitions. At the same time, one can use the bivariate RGARCH model for

\footnotetext{
${ }^{7} \mathrm{~A}$ similar iteration is performed in the case of the GJR-GARCH model.
} 
forecasting. In this case one obtains one-period-ahead forecasts of the full covariance matrix by iterating forward on (13).

If one is interested instead in multi-period forecasts, some adjustments are necessary. Within the "GRAS" copula specification, one would need to model the dynamics of the realized measure of correlation, for example with a heterogeneous autoregressive model à la Audrino and Corsi (2010), in order to construct multiperiod forecasts of it. The time-varying correlation coefficient of the Student copula results then immediately from (11) with the realized measure replaced by its forecast and by the nullity of the multi-period score of the copula likelihood. Second, to predict the variances instead of the log-variances, one should proceed by residual bootstrap based on (8) and (9), as indicated for example in Lunde and Olesen (2013), see also Hansen et al. (2014). The variance forecasts will be given by the average of the exponential of all the possible log-variance forecasts obtained in $S$ simulations. A similar bootstrap procedure, but involving (13), (14) and (15), easily produces covariance forecasts as for the bivariate model in Dumitrescu and Hansen (2018).

Once the forecast sequences on the covariance matrix are obtained, they are compared based on the robust loss function proposed by Patton (2011) and the Model Confidence Set (MCS) test introduced by Hansen et al. (2011). More specifically, in order to compare the covariance matrix forecasts, one is supposed to use a loss function defined as a general function of the covariance forecasts and the true covariances. But the true volatilities and correlations are unobserved and forecast evaluation has to rely on an ex-post covariance estimator generally called "proxy". To this aim, both the realized covariance and the multivariate realized kernel estimators are used as proxies.

However, it is well known that, because of its randomness, the use of a proxy may distort the ranking of models based on loss functions. Andersen et al. (2005) show that the comparison of losses - even based on a conditionally unbiased proxy - 
may lead to a different ranking than the one obtained if the true latent variable had been used. More recently, Hansen and Lunde (2006), Patton and Sheppard (2009a), Patton (2011), Laurent et al. (2013) have also focused on the possible distortions induced by the use of a noisy proxy in the ranking of variance/volatility forecasts. In the context of univariate models, Hansen and Lunde (2006) provided conditions for both the loss function and the volatility proxy under which the approximated ranking (based on the proxy) is consistent with the true ranking (based on the unobserved volatility). ${ }^{8}$ Going further, Patton (2011) derives necessary and sufficient conditions on the functional form of the loss function for the models to be consistently ordered. Laurent et al. (2013) extended the previous results to the evaluation of multivariate volatility models, the comparison and the ordering relying on the sequences of covariance matrices. The authors provide (i) the theoretical conditions that a loss function should satisfy in order to deliver the same ranking regardless whether the true conditional variance matrix or an unbiased proxy of it is used, and (ii) the necessary and sufficient conditions on the functional form of the loss function to order the models consistently in matrix and vector spaces.

Following these studies, we consider as loss functions the quasi-likelihood loss function $(Q L i k e)$, the squared Frobenius distance and the EntryWise norm:

$$
\begin{aligned}
\operatorname{QLike}\left(\Sigma_{t}, H_{t}\right) & =\operatorname{tr}\left(H_{t}^{-1} \hat{\Sigma}_{t}\right)-\log \left|H_{t}^{-1} \hat{\Sigma}_{t}\right|-K, \\
L_{F}\left(\Sigma_{t}, H_{t}\right) & =\operatorname{tr}\left[\left(\hat{\Sigma}_{\mathrm{t}}-\mathrm{H}_{\mathrm{t}}\right)^{2}\right]=\sum_{N} \lambda_{i}, \\
L_{1 M}\left(\Sigma_{t}, H_{t}\right) & =\operatorname{tr}\left[\left|\hat{\Sigma}_{\mathrm{t}}-\mathrm{H}_{\mathrm{t}}\right|\right],
\end{aligned}
$$

\footnotetext{
${ }^{8}$ At this stage, a confusion between the consistency of the ranking and the invariance of the ordering may appear and it has to be avoided. As stated by Laurent et al. (2013), consistency is understood as the accuracy of the proxy and this for a given loss function, i.e. we talk about consistency between the true and the approximated ranking. On the other hand, the invariance of the ranking means that the ordering does not change with respect to the choice of the loss function.
} 
where $\hat{\Sigma}_{t}$ is a realized proxy of the true but latent conditional covariance matrix, $H_{t}$ is a candidate model for the conditional covariance matrix and $\lambda_{i}$ are the positive eigenvalues of the matrix $\left(\hat{\Sigma}_{t}-H_{t}\right)^{2}$.

Knowing that in the empirical application we have to compare six competing models in terms of their predictive abilities, we decide to implement the Model Confidence Set approach, which is a multiple comparison-based test proposed by Hansen et al. (2011). This test aims at identifying, among the set of competing models, $M_{0}$, the subset of models $\hat{M}_{1-\alpha}$, which are equivalent in terms of forecasting ability and which outperform all the other models for a given confidence level (1 a). ${ }^{9}$ We set the significance level for the MCS to $\alpha=\{10 \%, 25 \%\}$ and use 10,000 bootstrap resamples (with a block length of 12 daily observations) to obtain the distribution under the null of equal predictive accuracy.

\section{Empirical Application}

In this section we implement the two types of multivariate models including realized measures that were discussed in Section 2.2.2, and analyze their forecasting abilities relative to those of a selection of benchmark models.

\subsection{Dataset}

We use an extensive sample of high-frequency 1-minute data on S\&P 500 companies from January 5, 2009 to June 30, 2016. The data are quotations prices taken from QuantQuote. All high-frequency prices are cleaned using the procedure outlined in Barndorff-Nielsen et al. (2009), and public holidays (e.g. Christmas, New Year, Good

\footnotetext{
${ }^{9}$ The main advantages of the MCS test are that we do not have to specify a benchmark model and that it relies on simple hypotheses that lead to standard asymptotics. However, when the number of competing models is large, the test statistic has a non-standard distribution, reason for which a bootstrap scheme is employed to obtain the distribution under the null hypothesis.
} 
Friday, Easter Monday, Memorial Day, US National Day, Labor Day, Thanksgiving) are deleted. The daily returns are constructed as the log-difference of close prices, i.e. the last intraday price observed before or at 4PM precisely. The realized measures of volatility and correlation are computed from 390 one-minute intra-day returns using the multivariate realized kernel estimator by Barndorff-Nielsen et al. (2011). This estimator is widely used nowadays, since it is robust to microstructure noise and it insures the positive definiteness of the estimated covariance matrix. We hence rely on it for both the estimation of the model and the evaluation of its forecasting abilities. The Realized Covariance estimator of Andersen et al. (2003) is also used as a covariance matrix proxy in the spirit of a robustness check, see Section 2.1 for a short description of both realized measures.

We consider three pairs of stocks in this analysis: Citigroup - JP Morgan (C JPM), Boeing - Procter\&Gamble (Boeing - PG), and Pepsi - Coca-Cola (PEP - KO). This selection covers assets from different sectors and different levels of sample linear correlation: around $0.66,0.38$, and 0.60 , respectively. Such an analysis is expected to provide empirical evidence on the models' forecasting abilities according to the level of dependence between the companies and their sector of activity. Table 1 presents a summary of descriptive statistics for the daily log-return series, the Realized Kernel measure and the Realized Correlation estimator used in our analysis. The daily returns exhibit very small means for all assets and quite large overdispersion (i.e. the standard deviation is greater than the mean). They also display a high kurtosis, which indicates much heavier tails than a normal distribution. The skewness of the return series of the couple Citigroup - JP Morgan is positive, while the other return series have a left-skewed shape. Citigroup appears as the most volatile asset (having a standard deviation of 5.049 and a Realized Kernel average of 6.192), followed by Boeing. As expected, the realized kernel series present very strong positive skew and kurtosis. At the same time, the realized correlations have a positive mean and they 
are negatively skewed.

Table 1: Descriptive statistics

\begin{tabular}{|c|c|c|c|c|c|c|c|c|c|}
\hline & C-JPM & & & & & & & & \\
\hline \multirow{3}{*}{ Returns } & & Min & Q25\% & Median & Mean & Q75\% & Std Dev & Skewness & Kurtosis \\
\hline & $\mathrm{C}$ & -40.23 & -1.586 & 0.000 & -0.030 & 1.410 & 5.049 & 0.026 & 10.10 \\
\hline & JPM & -4.549 & -0.360 & 0.000 & 0.009 & 0.361 & 0.759 & 0.111 & 8.131 \\
\hline \multirow[t]{2}{*}{ RK } & $\mathrm{C}$ & 0.101 & 0.908 & 2.034 & 6.192 & 4.638 & 25.04 & 18.90 & 538.4 \\
\hline & JPM & 0.060 & 0.873 & 1.869 & 4.493 & 4.273 & 10.55 & 10.74 & 184.6 \\
\hline \multirow[t]{3}{*}{ RCorr } & & -0.341 & 0.453 & 0.597 & 0.573 & 0.721 & 0.197 & -0.688 & 3.438 \\
\hline & Boeing-PG & & & & & & & & \\
\hline & & Min & Q25\% & Median & Mean & Q75\% & Std Dev & Skewness & Kurtosis \\
\hline \multirow[t]{2}{*}{ Returns } & Boeing & -11.23 & -0.502 & 0.024 & 0.021 & 0.554 & 1.079 & -0.433 & 10.35 \\
\hline & PG & -8.954 & -0.260 & 0.016 & 0.013 & 0.301 & 0.571 & -1.018 & 21.00 \\
\hline \multirow[t]{2}{*}{ RK } & Boeing & 0.073 & 0.865 & 1.559 & 2.680 & 3.042 & 3.788 & 5.829 & 56.95 \\
\hline & PG & 0.027 & 0.436 & 0.758 & 1.544 & 1.594 & 3.017 & 11.49 & 229.0 \\
\hline \multirow[t]{3}{*}{ RCorr } & & -0.608 & 0.112 & 0.264 & 0.261 & 0.419 & 0.221 & -0.168 & 2.765 \\
\hline & PEP-CO & & & & & & & & \\
\hline & & Min & Q25\% & Median & Mean & Q75\% & Std Dev & Skewness & Kurtosis \\
\hline \multirow[t]{2}{*}{ Returns } & PEP & -6.071 & -0.293 & 0.008 & 0.018 & 0.325 & 0.584 & -0.186 & 9.072 \\
\hline & $\mathrm{CO}$ & -2.432 & -0.142 & 0.004 & 0.005 & 0.156 & 0.312 & -0.229 & 8.053 \\
\hline \multirow[t]{2}{*}{ RK } & PEP & 0.034 & 0.427 & 0.799 & 1.662 & 1.775 & 3.567 & 22.38 & 884.5 \\
\hline & $\mathrm{CO}$ & 0.047 & 0.453 & 0.817 & 1.566 & 1.709 & 2.420 & 6.848 & 90.83 \\
\hline RCorr & & -0.478 & 0.305 & 0.478 & 0.455 & 0.629 & 0.227 & -0.464 & 2.821 \\
\hline
\end{tabular}

Note: The table displays the summary statistics on daily log-returns (Returns), Realized Kernel (RK) and Realized Correlation (RCorr) for the six U.S. companies under analysis. Min stands for the minimum value, $\mathrm{Q} \alpha \%$ for the $\alpha$ quantile of the distribution and StdDev for the standard deviation.

One-period ahead forecasts are obtained for the period July 2014 to June 2016 through the rolling windows strategy with daily re-estimation of the competing models on 5 years and 6 months of data each time. This amounts to an in-sample size of 1341 observations and 499 out-of sample observations on which we base the performance evaluation tests. 


\subsection{Preliminary Results}

This analysis is designed to select the best copula functional form and to check whether simplifying the specification of the multivariate RGARCH model could be useful. It is based on likelihood comparisons, where the out-of-sample likelihood is obtained simply by plugging the in-sample estimates into the out-of-sample loglikelihood function. Note that in both analyses we report the likelihood and not the likelihood ratios for two reasons: i) in the QMLE framework, the likelihood-ratio statistic does not have a standard $\chi^{2}$ limit distribution, and ii) the asymptotic distribution of the out-of-sample likelihood-ratio statistic is non-standard (see Hansen et al., 2012, for a more thorough discussion on this topic).

Table 2 reports the in-sample and pseudo-out-of-sample likelihood for a large number of constant and time-varying copulas conditional on the three types of marginal models selected, i.e. GJR-GARCH, RGARCH and RRGARCH. The results generally reveal the improved fit provided by the time-varying Student's tGRAS copula, both in-sample and out-of-sample, regardless of the asset-pair under analysis. This finding indicates that allowing for joint fat tails in multivariate models of asset returns is important and more relevant than accounting for asymmetry for example with a Rotated Gumbel-GAS copula. The superior likelihood of the GRAS specification of this copula observed in most of the cases analyzed also shows that high-frequency data on correlations seems to improve the fit of dynamic copula models both in-sample and out-of-sample.

At the same time, Table 3 presents full and partial likelihood results for two specifications of our bivariate RGARCH model, i.e. the full and the diagonal specifications described in Section 2.3. Recall that the diagonal model assumes that $\beta$ is a diagonal matrix, i.e. there are no direct spillovers between the elements of the assets' conditional covariance matrix. The results provide evidence of possible out- 
of-sample gains from simplifying the structure of the model and support the use of the diagonal specification in the subsequent forecasting analysis.

Nevertheless, this preliminary analysis is just indicative and it does not distinguish between the performance of the selected (R)RGARCH t-GRAS copula models, that of the diagonal bivariate RGARCH model and the performance of the traditional benchmarks. Therefore, in the following, we specifically check the relative out-of-sample forecasting performance of the selected multivariate models.

\subsection{Forecast Evaluation}

As discussed previously, the empirical application illustrates the results for oneperiod-ahead forecasts on the conditional covariance matrix obtained from six competing models. Their forecasting abilities are compared based on the MCS test of Hansen et al. (2011) with three robust loss functions proposed by Patton (2011), i.e., the quasi-likelihood loss function, the squared Frobenius distance and the EntryWise matrix norm. Indeed, simulation results in Patton and Sheppard (2009a), and empirical results in Hansen and Lunde (2005), Patton and Sheppard (2009b) and Patton and Sheppard (2015) all suggest that using such loss functions leads to good power to reject inferior estimators when the true variance (covariance matrix) is unobserved and hence replaced by an unbiased proxy.

The results are synthesized in Tables 4 and 5. They display the average loss over the evaluation period and the MCS p-value for each competing model under analysis. The three aforementioned robust loss functions are considered for each couple of stocks, i.e., Citigroup - JP Morgan, Boeing - Procter\&Gamble, and Pepsi - Coca-Cola. The best models, as identified by the MCS test, are signaled by one (significance level of 10\%) and two (significance level of 25\%) asterisks, respectively. The main difference between these two tables is that they rely on different covari- 
ance matrix proxies for the true unobserved covariance matrix when evaluating the forecasting abilities of the six models. Table 4 uses the multivariate realized kernel of Barndorff-Nielsen et al. (2011), while the results in Table 5 rely on the classic realized covariance of Andersen et al. (2003).

Taking as example the couple Citigroup - JP Morgan (Table 4), we observe that the smallest average QLIKE losses are recorded by the bivariate RGARCH model, followed by the RRGARCH-GRAS copula and the RGARCH-GRAS copula specifications. Only the first specification is selected as the best model by the MCS test. The two other specifications are in the subset of superior forecasting models when the MCS test is based on the losses given by the squared Frobenius distance. When considering the EntryWise norm, the MCS test identifies RRGARCH-GRAS copula as the best model. This change in the composition of the subset of best models according to the MCS is not surprising since the consistency of the ranking is defined with respect to a given loss function. Thus, the ranking may change when a different loss function is used in MCS. Identical results are obtained when changing the proxy used for the covariance matrix (see Table 5).

For the Boeing - Procter\&Gamble pair, the MCS test selects the GJR-GRAS copula and/or the GJR-GAS copula specifications as the subset of best models, whatever the loss function considered. Pepsi - Coca-Cola is the only couple including the traditional cDCC model in the subset of superior forecasting models among some other models.

All in all, these findings indicate that the new specifications making use of expost information contained in realized correlation data (either taking the form of multivariate realized models or copula-type approaches) lead to significant improvement in the quality of the conditional covariance (and implicitly, of the correlation) matrix forecasts. Most of the time, the use of realized measures of volatility also proves to be useful in the context of covariance forecasting, suggesting that both 
high-frequency-based measures of volatility and of correlation should be exploited to improve conditional covariance forecasts in larger-scale empirical analyses.

\section{Conclusion}

In this paper we tackle the usefulness of ex-post high-frequency-based measures of volatility and correlation in forecasting the conditional covariance matrix. For this, we propose various model specifications that rely on the multivariate RGARCH framework, or on univariate (R)RGARCH / GJR-GARCH models augmented with copula dependence functions. These models hence include either realized measures of variance or realized measures of correlation, or both or none. A horse-race of their out-of-sample forecasting abilities is organized in an empirical analysis on three pairs of assets, i.e., Citigroup - JP Morgan, Boeing - Procter\&Gample, and Pepsi - CocaCola, that exhibit different levels of unconditional correlation and cover different sectors. The one-period-ahead forecasting abilities of the six models in competition are scrutinized through the MCS test by relying on robust loss functions. Our main finding is that the specifications proposed contain useful information in covariance forecasting that goes beyond that of traditional multivariate GARCH models. Still, none of the models proposed outperforms all the other specifications in all cases. We thus show that the role the realized measures of volatility and correlation play in conditional covariance forecasting seems to deserve more attention in larger-scale empirical forecasting analyses. Besides, the possible connection between the results of the forecast evaluation and the level of correlation between the assets, as indicated by Pearson's coefficient, is still to be investigated, as no clear pattern emerges from our analysis. 


\section{References}

G. P. Aielli. Dynamic conditional correlation: On properties and estimation. Journal of Business \& Economic Statistics, 31(3):282-299, 2013.

Y. Aït-Sahalia, P. A. Mykland, and L. Zhang. How often to sample a continuous-time process in the presence of market microstructure noise. The review of financial studies, 18(2):351-416, 2005.

T. G. Andersen and T. Bollerslev. Answering the skeptics: Yes, standard volatility models do provide accurate forecasts. International Economic Review, 39(4):885$905,1998$.

T. G. Andersen, T. Bollerslev, F. X. Diebold, and P. Labys. The distribution of exchange rate volatility. Journal of the American Statistical Association, 96(453): 42-55, 2001. Correction published in 2003, volume 98, page 501.

T. G. Andersen, T. Bollerslev, F. X. Diebold, and P. Labys. Modeling and forecasting realized volatility. Econometrica, 71(2):579-625, 2003.

T. G. Andersen, T. Bollerslev, and N. Meddahi. Correcting the errors: Volatility forecast evaluation using high-frequency data and realized volatilities. Econometrica, 73(1):279-296, 2005.

T. G. Andersen, D. Dobrev, and E. Schaumburg. Duration-based volatility estimation. Working paper, 2009.

T. G. Andersen, D. Dobrev, and E. Schaumburg. Jump-robust volatility estimation using nearest neighbor truncation. Journal of Econometrics, 169(1):75-93, 2012. 
E. Andreou and E. Ghysels. Rolling-sample volatility estimators: Some new theoretical, simulation, and empirical results. Journal of Business 8 Economic Statistics, 20(3):363-376, 2002.

F. Audrino and F. Corsi. Modeling tick-by-tick realized correlations. Computational Statistics \& Data Analysis, 54(11):2372-2382, 2010.

X. Bai, J. R. Russell, and G. C. Tiao. Effects of non-normality and dependence on the precision of variance estimates using high-frequency financial data. Working paper, University of Chicago, GSB, 2004.

D. Banulescu-Radu, C. Hurlin, B. Candelon, and S. Laurent. Do we need high frequency data to forecast variances? Annals of Economics and Statistics/Annales d'Économie et de Statistique, (123/124):135-174, 2016.

D. Banulescu-Radu, P. R. Hansen, Z. Huang, and M. Matei. Volatility during the financial crisis through the lens of high frequency data: A realized GARCH approach. Working paper, SSRN, 2018.

O. E. Barndorff-Nielsen and N. Shephard. Econometric analysis of realised volatility and its use in estimating stochastic volatility models. Journal of the Royal Statistical Society B, 64(2):253-280, 2002.

O. E. Barndorff-Nielsen and N. Shephard. Power and bipower variation with stochastic volatility and jumps (with discussion). Journal of Financial Econometrics, 2 (1):1-48, 2004.

O. E. Barndorff-Nielsen, P. R. Hansen, A. Lunde, and N. Shephard. Designing realized kernels to measure the ex post variation of equity prices in the presence of noise. Econometrica, 76(6):1481-1536, 2008. 
O. E. Barndorff-Nielsen, P. R. Hansen, A. Lunde, and N. Shephard. Realized kernels in practice: Trades and quotes. The Econometrics Journal, 12(3):C1-C32, 2009.

O. E. Barndorff-Nielsen, P. R. Hansen, A. Lunde, and N. Shephard. Multivariate realised kernels: consistent positive semi-definite estimators of the covariation of equity prices with noise and non-synchronous trading. Journal of Econometrics, 162(2):149-169, 2011.

L. Bauwens, C. M. Hafner, and S. Laurent. Handbook of volatility models and their applications, volume 3. John Wiley \& Sons, 2012.

L. A. Callot, A. B. Kock, and M. C. Medeiros. Modeling and forecasting large realized covariance matrices and portfolio choice. Journal of Applied Econometrics, 32(1): 140-158, 2017.

R. Chiriac and V. Voev. Modelling and forecasting multivariate realized volatility. Journal of Applied Econometrics, 26(6):922-947, 2011.

K. Christensen and M. Podolskij. Realized range-based estimation of Integrated Variance. Journal of Econometrics, 141(2):323-349, 2007.

K. Christensen, R. Oomen, and M. Podolskij. Realised quantile-based estimation of the integrated variance. Journal of Econometrics, 159(1):74-98, 2010.

F. Corsi. A simple approximate long-memory model of realized volatility. Journal of Financial Econometrics, 7(2):174-196, 2009.

D. D. Creal, S. J. Koopman, and A. Lucas. Generalized autoregressive score models with applications. Journal of Applied Econometrics, 28(5):777-795, 2013.

M. M. Dacorogna, R. Gencay, U. Müller, R. B. Olsen, and O. V. Pictet. An introduction to high-frequency finance. Academic Press, London, 2001. 
E.-I. Dumitrescu and P. R. Hansen. Exchange rate volatility forecasting: a multivariate realized-GARCH approach. Working Paper, 2018.

R. F. Engle. New frontiers for ARCH models. Journal of Applied Econometrics, 17 (5):425-446, 2002.

R. F. Engle and G. M. Gallo. A multiple indicators model for volatility using intradaily data. Journal of Econometrics, 131(1-2):3-27, 2006.

R. F. Engle and K. F. Kroner. Multivariate simultaneous generalized ARCH. Econometric theory, 11(1):122-150, 1995.

S. Gonçalves and H. White. Maximum likelihood and the bootstrap for nonlinear dynamic models. Journal of Econometrics, 119(1):199-219, 2004.

J. E. Griffin and R. C. Oomen. Covariance measurement in the presence of nonsynchronous trading and market microstructure noise. Journal of Econometrics, 160(1):58-68, 2011.

P. R. Hansen and G. Horel. Quadratic variation by markov chains. Working paper, 2009.

P. R. Hansen and Z. Huang. Exponential GARCH modeling with realized measures of volatility. Journal of Business $\mathcal{G}$ Economic Statistics, 34(2):269-287, 2016.

P. R. Hansen and A. Lunde. A forecast comparison of volatility models: does anything beat a GARCH (1,1)? Journal of applied econometrics, 20(7):873-889, 2005.

P. R. Hansen and A. Lunde. Consistent ranking of volatility models. Journal of Econometrics, 131(1-2):97-121, 2006.

P. R. Hansen, A. Lunde, and J. M. Nason. The model confidence set. Econometrica, 79(2):456-497, 2011. 
P. R. Hansen, Z. Huang, and H. H. Shek. Realized GARCH: a joint model for returns and realized measures of volatility. Journal of Applied Econometrics, 27 (6):877-906, 2012.

P. R. Hansen, A. Lunde, and V. Voev. Realized beta GARCH: A multivariate GARCH model with realized measures of volatility. Journal of Applied Econometrics, 29(5):774-799, 2014.

A. C. Harvey. Dynamic models for volatility and heavy tails: With applications to financial and economic time series. Cambridge University Press, 2013.

J. Jacod, Y. Li, P. A. Mykland, M. Podolskij, and M. Vetter. Microstructure noise in the continuous case: the pre-averaging approach. Stochastic Processes and their Applications, 119(7):2249-2276, 2009.

S. Laurent, J. V. Rombouts, and F. Violante. On loss functions and ranking forecasting performances of multivariate volatility models. Journal of Econometrics, 173(1):1-10, 2013.

L. Y. Liu, A. J. Patton, and K. Sheppard. Does anything beat 5-minute rv? a comparison of realized measures across multiple asset classes. Journal of Econometrics, 187(1):293-311, 2015.

A. Lunde and K. V. Olesen. Modeling and forecasting the volatility of energy forward returns. Working Paper, 2013.

C. Mancini. Disentangling the jumps of the diffusion in a geometric jumping brownian motion. Giornale dell'Istituto Italiano degli Attuari, 64(19-47):44, 2001.

C. Mancini. Non-parametric threshold estimation for models with stochastic diffusion coefficient and jumps. Scandinavian Journal of Statistics, 36(2):270-296, 2009. 
M. Martens and D. Van Dijk. Measuring volatility with the realized range. Journal of Econometrics, 138(1):181-207, 2007.

A. Patton. Copula methods for forecasting multivariate time series. In Handbook of economic forecasting, volume 2, pages 899-960. Elsevier, 2013.

A. J. Patton. Estimation of multivariate models for time series of possibly different lengths. Journal of Applied Econometrics, 21(2):147-173, 2006.

A. J. Patton. Volatility forecast comparison using imperfect volatility proxies. Journal of Econometrics, 160(1):246-256, 2011.

A. J. Patton. A review of copula models for economic time series. Journal of Multivariate Analysis, 110:4-18, 2012.

A. J. Patton and K. Sheppard. Evaluating volatility and correlation forecasts. In Handbook of financial time series, pages 801-838. Springer, 2009a.

A. J. Patton and K. Sheppard. Optimal combinations of realised volatility estimators. International Journal of Forecasting, 25(2):218-238, 2009b.

A. J. Patton and K. Sheppard. Good volatility, bad volatility: Signed jumps and the persistence of volatility. Review of Economics and Statistics, 97(3):683-697, 2015.

M. Podolskij and M. Vetter. Bipower-type estimation in a noisy diffusion setting. Stochastic processes and their applications, 119(9):2803-2831, 2009.

I. D. L. Salvatierra and A. J. Patton. Dynamic copula models and high-frequency data. Journal of Empirical Finance, 30:120-135, 2015.

N. Shephard and K. Sheppard. Realising the future: forecasting with high-frequencybased volatility (HEAVY) models. Journal of Applied Econometrics, 25(2):197231, 2010 . 
M. Sklar. Fonctions de répartition à $n$ dimensions et leurs marges. Université Paris 8, 1959.

H. White. Estimation, inference and specification analysis. Number 22. Cambridge University Press, 1996.

L. Zhang. Efficient estimation of stochastic volatility using noisy observations: A multi-scale approach. Bernoulli, 12(6):1019-1043, 2006.

L. Zhang, P. Mykland, and Y. Ait-Sahalia. A tale of two time scales. Journal of the American Statistical Association, 100(472):1394-1411, 2005.

\section{Appendix: Tables and Figures}




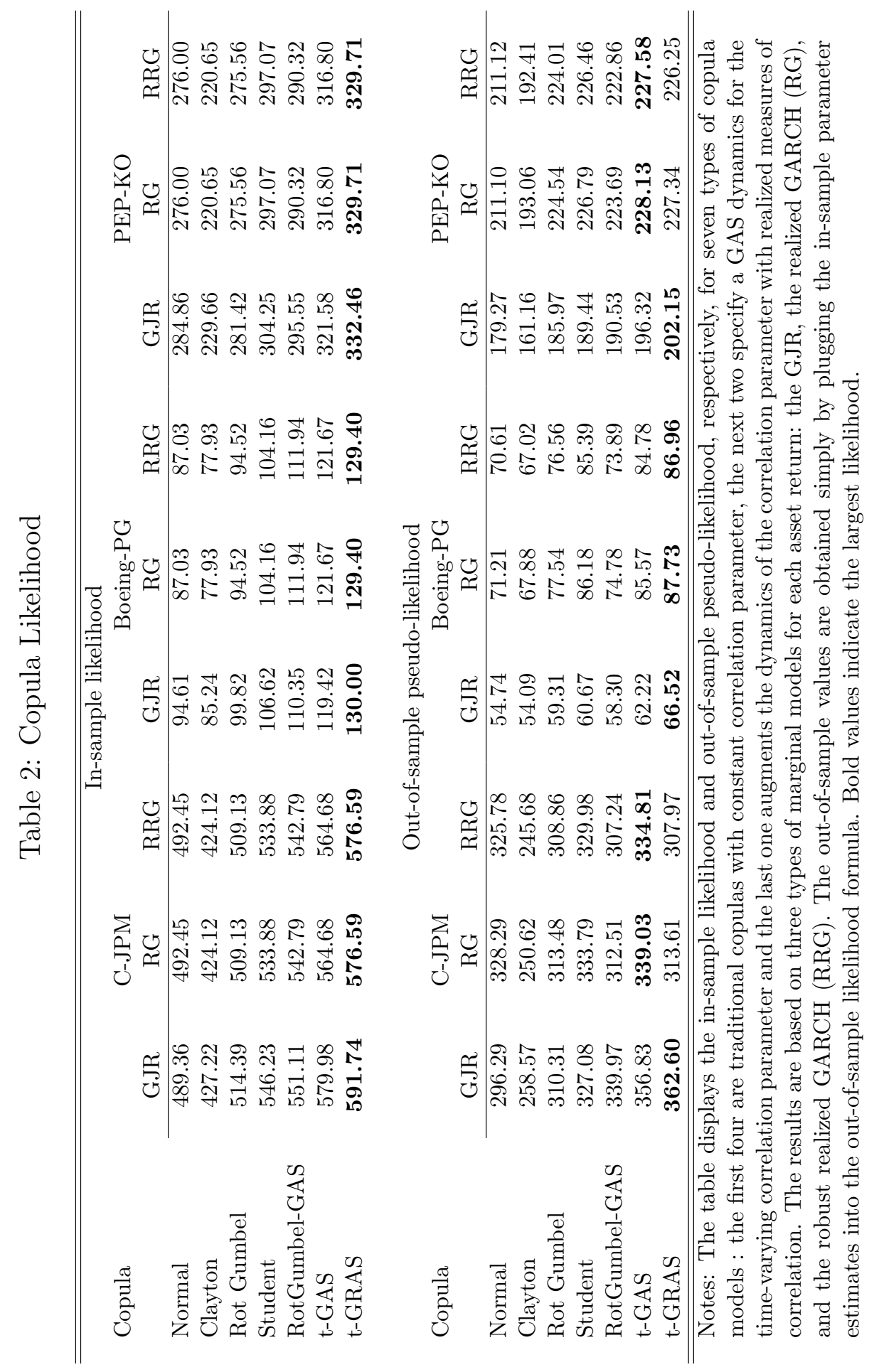


Table 3: Bivariate Realized GARCH likelihood

\begin{tabular}{lccc}
\hline \hline & \multicolumn{2}{c}{ In-sample likelihood } \\
& C-JPM & Boeing-PG & PEP-KO \\
\cline { 2 - 4 } Diagonal Bivariate & $\mathbf{- 5 2 3 6 . 7 1}$ & $\mathbf{- 6 2 6 6 . 4 5}$ & $\mathbf{- 3 5 1 3 . 2 1}$ \\
Full Bivariate & $\mathbf{- 4 6 6 9 . 3 6}$ & $\mathbf{- 5 0 5 5 . 6 2}$ & $\mathbf{- 2 7 4 2 . 9 9}$ \\
& & & \\
& Out-of-sample likelihood & \\
& C-JPM & Boeing-PG & PEP-KO \\
\cline { 2 - 4 } Diagonal Bivariate & $\mathbf{- 9 1 6 . 1 0}$ & $\mathbf{- 1 7 3 7 . 8 1}$ & $\mathbf{- 1 0 4 8 . 8 9}$ \\
Full Bivariate & -2004.80 & -2745.44 & -1629.99 \\
\hline \hline
\end{tabular}

Note: The table displays the in-sample likelihood and out-of-sample pseudo-likelihood, respectively, for the full and diagonal bivariate RGARCH models. The out-of-sample values are obtained by plugging the in-sample parameter estimates into the out-of-sample likelihood formula. Bold values indicate the largest likelihood, i.e. the preferred specification. 
Table 4: Out-of-sample forecast evaluation using Realized Kernel covariance proxy (MCS test)

\begin{tabular}{lcccccc}
\hline \hline & \multicolumn{9}{c}{ QLIKE } \\
& \multicolumn{2}{c}{ C-JPM } & \multicolumn{2}{c}{ Boeing-PG } & \multicolumn{2}{c}{ PEP-KO } \\
& Loss & $P_{M C S}$ & Loss & $P_{M C S}$ & Loss & $P_{M C S}$ \\
\hline Bivariate-RG & 0.82 & $1.00^{* *}$ & 3.77 & 0.00 & 5.82 & 0.00 \\
cDCC & 1.11 & 0.00 & 35.38 & 0.00 & 4.74 & $0.86^{* *}$ \\
RRG-GRAS-Copula & 0.88 & 0.03 & 3.49 & 0.00 & 6.46 & 0.00 \\
RG-GRAS-Copula & 0.90 & 0.00 & 3.47 & 0.00 & 6.47 & 0.00 \\
GJR-GRAS-Copula & 1.09 & 0.00 & 2.43 & 0.00 & 5.38 & 0.00 \\
GJR-GAS-Copula & 1.09 & 0.00 & 1.95 & $1.00^{* *}$ & 4.70 & $1.00^{* *}$
\end{tabular}

Squared Frobenius distance

\begin{tabular}{lcccccc} 
& \multicolumn{2}{c}{ C-JPM } & \multicolumn{2}{c}{ Boeing-PG } & \multicolumn{2}{c}{ PEP-KO } \\
& Loss & $P_{M C S}$ & Loss & $P_{M C S}$ & Loss & $P_{M C S}$ \\
\hline Bivariate-RG & 7.430 & $0.32^{* *}$ & 20.13 & 0.02 & 2.80 & $0.42^{* *}$ \\
cDCC & 14.47 & 0.01 & 21.18 & 0.01 & 2.81 & $0.55^{* *}$ \\
RRG-GRAS-Copula & 7.310 & $1.00^{* *}$ & 20.58 & 0.01 & 2.85 & $0.11^{*}$ \\
RG-GRAS-Copula & 7.330 & $0.32^{* *}$ & 20.56 & 0.01 & 2.83 & $0.10^{*}$ \\
GJR-GRAS-Copula & 15.94 & 0.02 & 19.80 & $1.00^{* *}$ & 2.78 & $0.55^{* *}$ \\
GJR-GAS-Copula & 15.94 & 0.02 & 19.80 & $0.14^{*}$ & 2.78 & $1.00^{* *}$
\end{tabular}

EntryWise norm

\begin{tabular}{lcccccc} 
& \multicolumn{2}{c}{ C-JPM } & \multicolumn{2}{c}{ Boeing-PG } & \multicolumn{2}{c}{ PEP-KO } \\
& Loss & $P_{M C S}$ & Loss & $P_{M C S}$ & Loss & $P_{M C S}$ \\
\hline Bivariate-RG & 1.93 & 0.04 & 3.65 & 0.00 & 1.46 & 0.00 \\
cDCC & 3.53 & 0.00 & 4.31 & 0.00 & 1.40 & $0.52^{* *}$ \\
RRG-GRAS-Copula & 1.84 & $1.00^{* *}$ & 3.69 & 0.00 & 1.47 & 0.00 \\
RG-GRAS-Copula & 1.85 & 0.04 & 3.69 & 0.00 & 1.46 & 0.00 \\
GJR-GRAS-Copula & 3.58 & 0.00 & 3.26 & $1.00^{* *}$ & 1.39 & $1.00^{* *}$ \\
GJR-GAS-Copula & 3.60 & 0.00 & 3.30 & 0.00 & 1.41 & 0.01
\end{tabular}

Note: The tables displays the MCS average loss over the evaluation sample and the pvalue for each of the six competing models under analysis. The conditional covariance forecasts in the $90 \%\left(\hat{M}_{90 \%}\right)$ and $75 \%\left(\hat{M}_{75 \%}\right)$ confidence sets are identified by one and two asterisks, respectively. Three loss functions are used, i.e., quasi-likelihood, the squared Frobenius distance and the EntryWise matrix norm. The multivariate realized kernel is used as a proxy for the true unobserved covariance matrix at each point in time. 
Table 5: Out-of-sample forecast evaluation using Realized covariance proxy (MCS test)

\begin{tabular}{lcccccc}
\hline \hline & \multicolumn{9}{c}{ QLIKE } \\
& \multicolumn{2}{c}{ C-JPM } & \multicolumn{2}{c}{ Boeing-PG } & \multicolumn{2}{c}{ PEP-KO } \\
& Loss & $P_{M C S}$ & Loss & $P_{M C S}$ & Loss & $P_{M C S}$ \\
\hline Bivariate-RG & 0.82 & $1.00^{* *}$ & 4.43 & 0.00 & 7.15 & 0.00 \\
cDCC & 1.05 & 0.00 & 36.66 & 0.00 & 5.80 & $0.90^{* *}$ \\
RRG-GRAS-Copula & 0.88 & 0.02 & 3.95 & 0.00 & 7.95 & 0.00 \\
RG-GRAS-Copula & 0.91 & 0.00 & 3.94 & 0.00 & 7.97 & 0.00 \\
GJR-GRAS-Copula & 1.04 & 0.00 & 2.95 & 0.00 & 6.59 & 0.00 \\
GJR-GAS-Copula & 1.03 & 0.00 & 2.28 & $1.00^{* *}$ & 5.76 & $1.00^{* *}$
\end{tabular}

Squared Frobenius distance

\begin{tabular}{lcccccc} 
& \multicolumn{2}{c}{ C-JPM } & \multicolumn{2}{c}{ Boeing-PG } & \multicolumn{2}{c}{ PEP-KO } \\
& Loss & $P_{M C S}$ & Loss & $P_{M C S}$ & Loss & $P_{M C S}$ \\
\hline Bivariate-RG & 8.100 & $0.30^{* *}$ & 7.35 & $0.10^{*}$ & 3.24 & 0.08 \\
cDCC & 14.37 & 0.01 & 8.440 & 0.00 & 3.24 & $0.59^{* *}$ \\
RRG-GRAS-Copula & 8.050 & $1.00^{* *}$ & 7.86 & 0.01 & 3.31 & 0.04 \\
RG-GRAS-Copula & 8.070 & $0.30^{* *}$ & 7.84 & 0.01 & 3.28 & 0.04 \\
GJR-GRAS-Copula & 15.73 & 0.02 & 7.08 & $0.18^{*}$ & 3.17 & $0.59^{* *}$ \\
GJR-GAS-Copula & 15.73 & 0.02 & 7.08 & $1.00^{* *}$ & 3.17 & $1.00^{* *}$
\end{tabular}

EntryWise norm

\begin{tabular}{lcccccc} 
& \multicolumn{2}{c}{ C-JPM } & \multicolumn{2}{c}{ Boeing-PG } & \multicolumn{2}{c}{ PEP-KO } \\
& Loss & $P_{M C S}$ & Loss & $P_{M C S}$ & Loss & $P_{M C S}$ \\
\hline Bivariate-RG & 1.78 & 0.04 & 3.68 & 0.00 & 1.64 & 0.00 \\
cDCC & 3.35 & 0.00 & 4.33 & 0.00 & 1.54 & $0.52^{* *}$ \\
RRG-GRAS-Copula & 1.74 & $1.00^{* *}$ & 3.71 & 0.00 & 1.64 & 0.00 \\
RG-GRAS-Copula & 1.75 & 0.04 & 3.70 & 0.00 & 1.64 & 0.00 \\
GJR-GRAS-Copula & 3.39 & 0.00 & 3.23 & $1.00^{* *}$ & 1.53 & $1.00^{* *}$ \\
GJR-GAS-Copula & 3.43 & 0.00 & 3.28 & 0.00 & 1.55 & 0.01
\end{tabular}

Note: The tables displays the MCS average loss over the evaluation sample and the pvalue for each of the six competing models under analysis. The conditional covariance forecasts in the $90 \%\left(\hat{M}_{90 \%}\right)$ and $75 \%\left(\hat{M}_{75 \%}\right)$ confidence sets are identified by one and two asterisks, respectively. Three loss functions are used, i.e., quasi-likelihood, the squared Frobenius distance and the EntryWise matrix norm. The realized covariance is used as a proxy for the true unobserved covariance matrix at each point in time. 